\title{
Evaluation of Contribution of Major Gaseous Pollutants in India with Implications on Health Scenario
}

\begin{abstract}
Life is the most essential entity in the World. What kind of world are we envisioning where we cannot breathe well due to pollution? One can have adverse consequences of being exposed to polluted air.Serious air contamination occurrences were watched often in north India, particularly at capital, Delhi. Dehradun, a capital of Uttarakhand, was amongst the $30^{\text {th }}$ most polluted cities as ranked by World Health Organisation (WHO). SO2 and NO2 are one of the contributing pollutants which are harmful to health. In this paper, the levels of both the pollutants are considered for various locations of Delhi and Dehradun, to knowwhothe major player is $\mathrm{SO} 2$ or $\mathrm{NO} 2$.
\end{abstract}

Akshara Pande, Kumud Pant, Akshita Rawat

Keywords: contamination, SO2, NO2, World Health Organisation.

\section{INTRODUCTION}

Air contamination ensues when dangerous or extreme amounts of substances are brought into Earth's air.In the past several years, the decline in air quality in cities and its direct impact on health and environment have been observed. World Health Organization (WHO) report showed around 4.2 million passing every year because of disclosure to ambient air pollution [1]. Exposure to polluted air for a while or longer can have terrible consequences over health.SO2 and $\mathrm{NO} 2$ are the components of the most noticeable concern.

One of the most polluted cities in the world are in India most notably New Delhi is known to be one such city with extreme levels of air pollutants. Dehradun the capital city of Uttarakhand is known for its scenic beauty and serene environment. Due to immense migration of people from different parts of India the once known heavenly and serene capital of Uttarakhand, Dehradun has seen alarming increase in levels of Sulphur Dioxide (SO2) and Nitrogen Dioxide (SO2) in few years. Realizing the need for analysis of varying levels of NO2 and SO2 in India the data for above two representative cities has been considered for further study.

\section{MATERIALS AND METHODS}

1. Kaggle- Falls under the Type Subsidiary of Google LLC. It is very useful repository for data scientists and machine learning experts as well as novices. The dataset used in this paper has been obtained from Kaggle at kaggle.com/datasets [2].

2. Microsoft excel- Has been used to plot 3D graphs for the data obtained from above.

3. Pairplot by Python libraries: The pairplot or scatter plot has been plotted for Exploratory Data Analysis (EDA) with the help of Python using Seaborn visualization library at python.org $[6,7]$.

\section{RESULT AND DISCUSSIONS}

The various areas of Dehradun considered for analysis were Clock Tower and ISBT in Dehradun. For Delhi Mayapuri Industrial area, Janakpuri and Nizamuddin were considered. The levels of both the pollutant gases were analyzed through Graphical analysis. University, Dehradun, India.

Akshita Rawat, Department of Life Sciences, Graphic Era Deemed to be University, Dehradun, India. 


\section{Clock Tower, Dehradun}

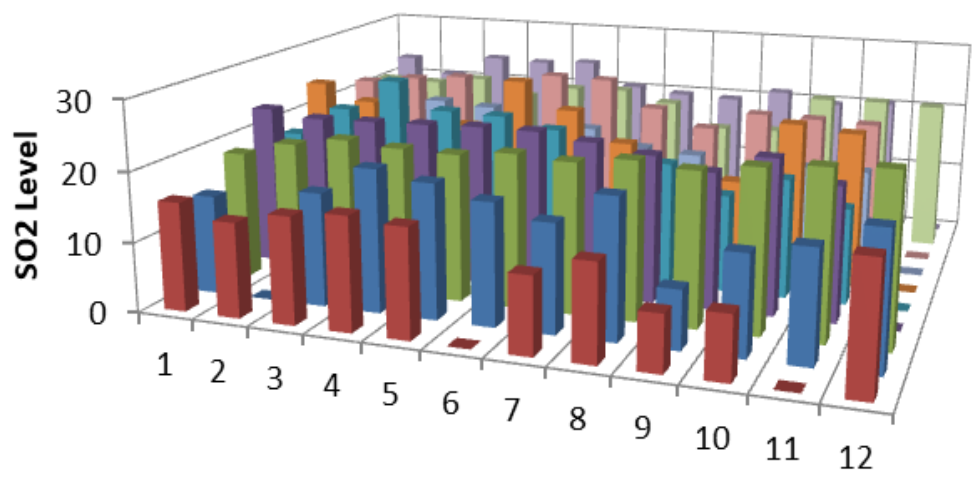

—SO2_2004

- SO2_2005

—SO2_2006

- SO2_2007

- SO2_2008

- SO2_2010

- SO2_2011

Months

—SO2_2012

\section{Figure 1: Variation in Sulphur Dioxide (SO2) level in Clock Tower, Dehradun from 2004 to 2012 as obtained from} Kaggle

Clock tower also known as GhantaGhar is a famous landmark of Dehradun. Located in the heart of Dehradun it is encircled by commercial complexes, shops and other places of interest. It remains buzzing with activity for twenty four (24) hours. As indicated in figure 1 the amount of Sulphur Dioxide (SO2) was maximum in 2007 and 2010. The least concentration was found in the year 2004the variation in level was minimum in 2004-5 with sudden increase in 2006. The trend has continued since then indicating gradual increase.
Figure 2 depicts variation inNitrogen Dioxide (NO2) level in Clock Tower, Dehradun from 2004 to 2015. The level of NO2 in June and November 2004 has found to be the minimum. The level of the gas has been found to be the maximum in the year 2007 and 2015. Since the data after these years was not available therefore just by looking at the trends it can be said that with the passage of time the level of NO2 is seeing a continuous trend.

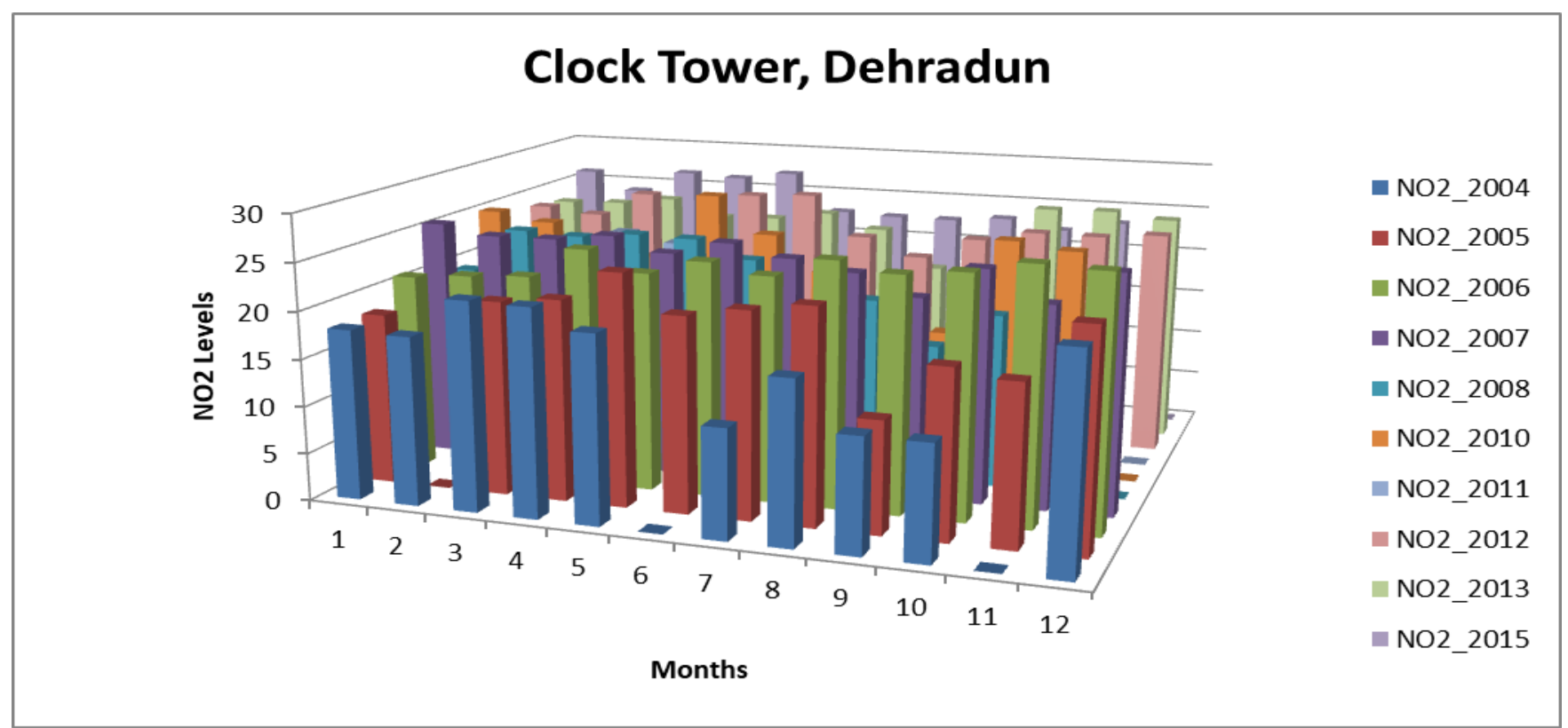

Figure 2: Variation in Nitrogen Dioxide (NO2) level in Clock Tower, Dehradun from 2004 to 2015 as obtained from Kaggle 


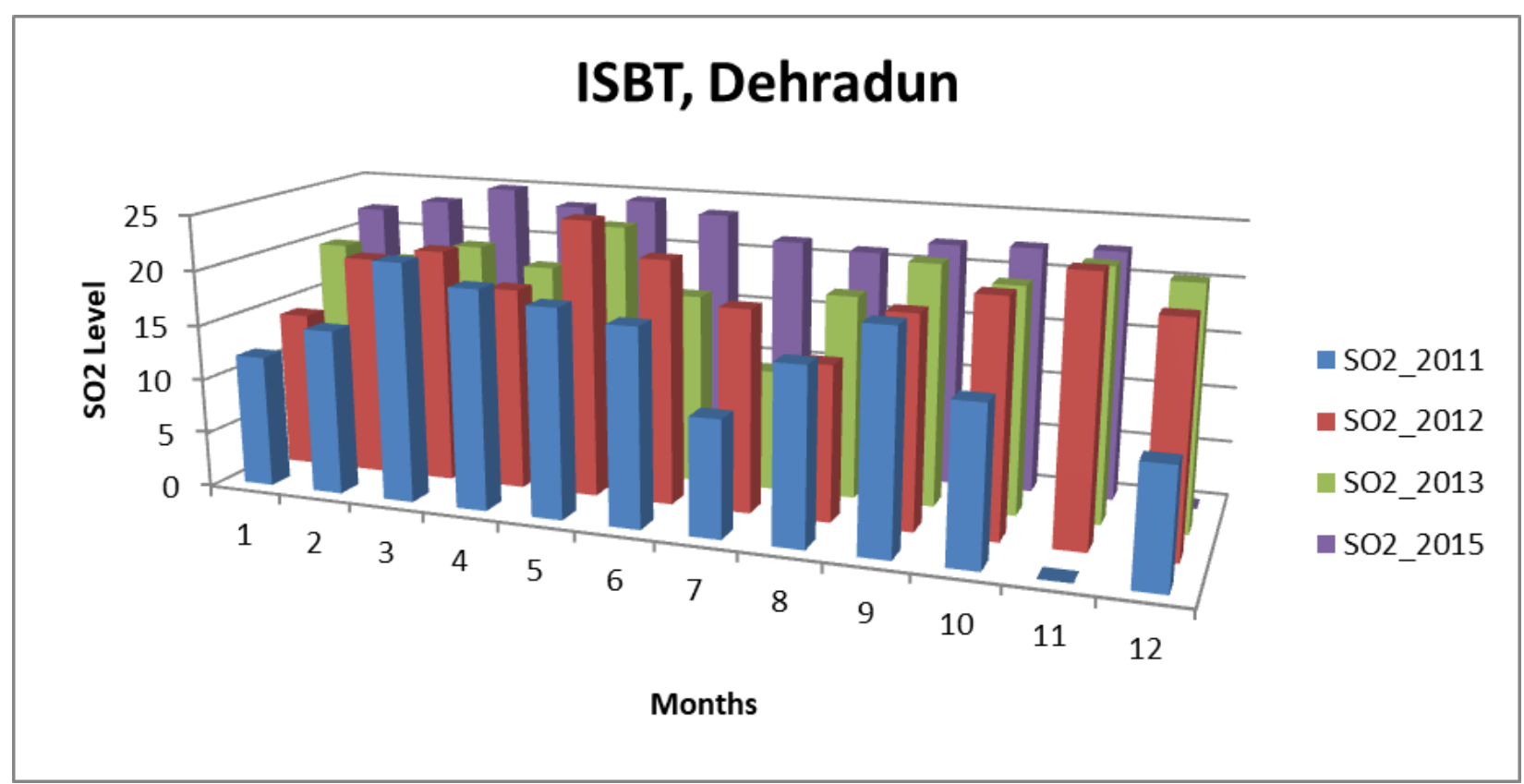

Figure 3: Variation in Sulphur Dioxide (SO2) level in ISBT, Dehradun from 2011 to 2015 as obtained from Kaggle

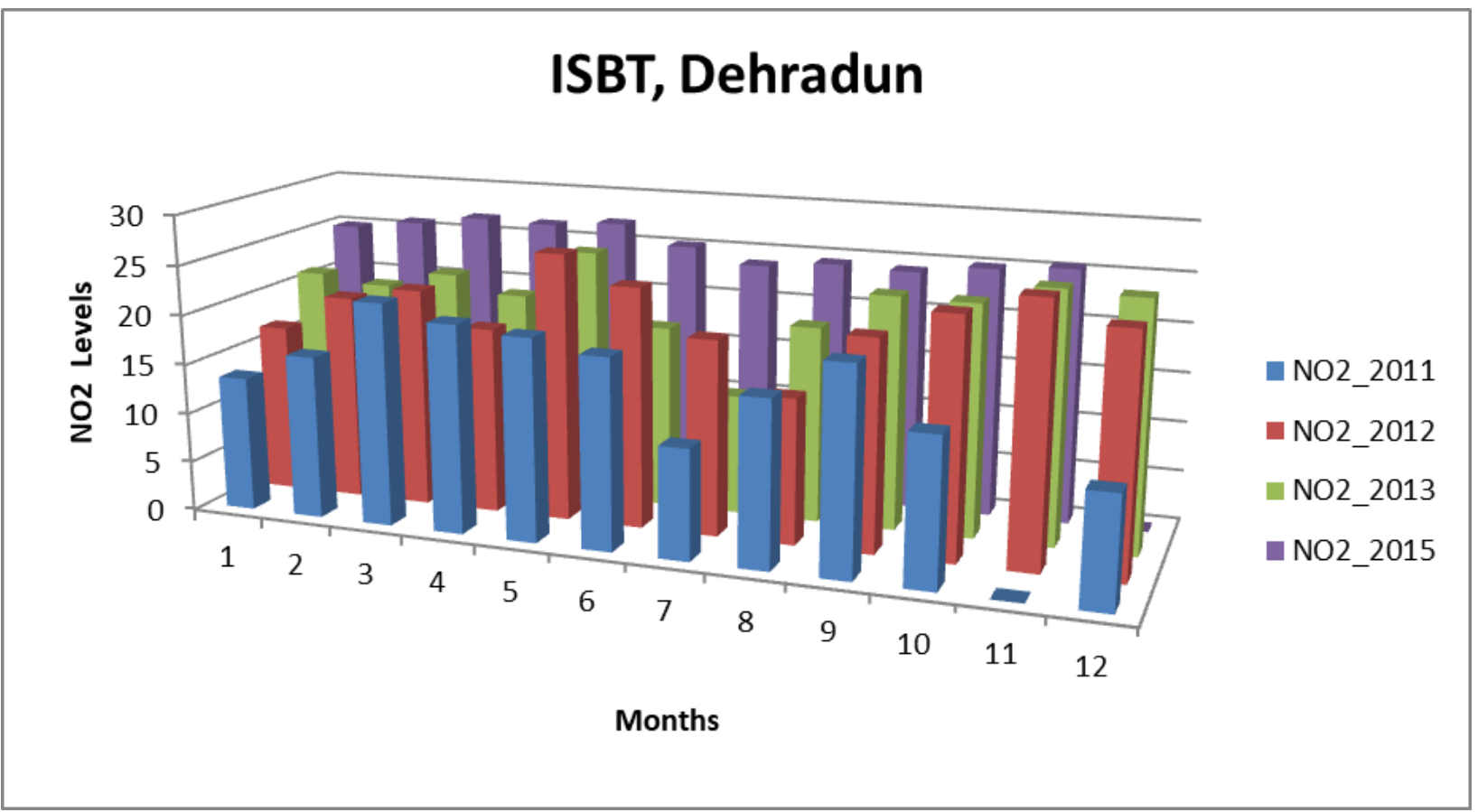

Figure 4: Variation in Nitrogen Dioxide (NO2) level in ISBT, Dehradun from 2011 to 2015 as obtained from Kaggle

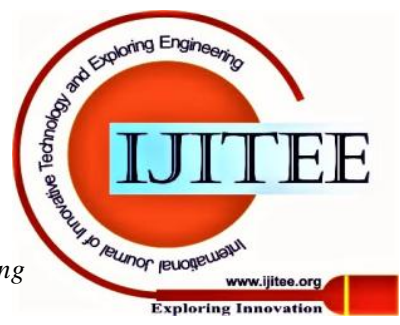




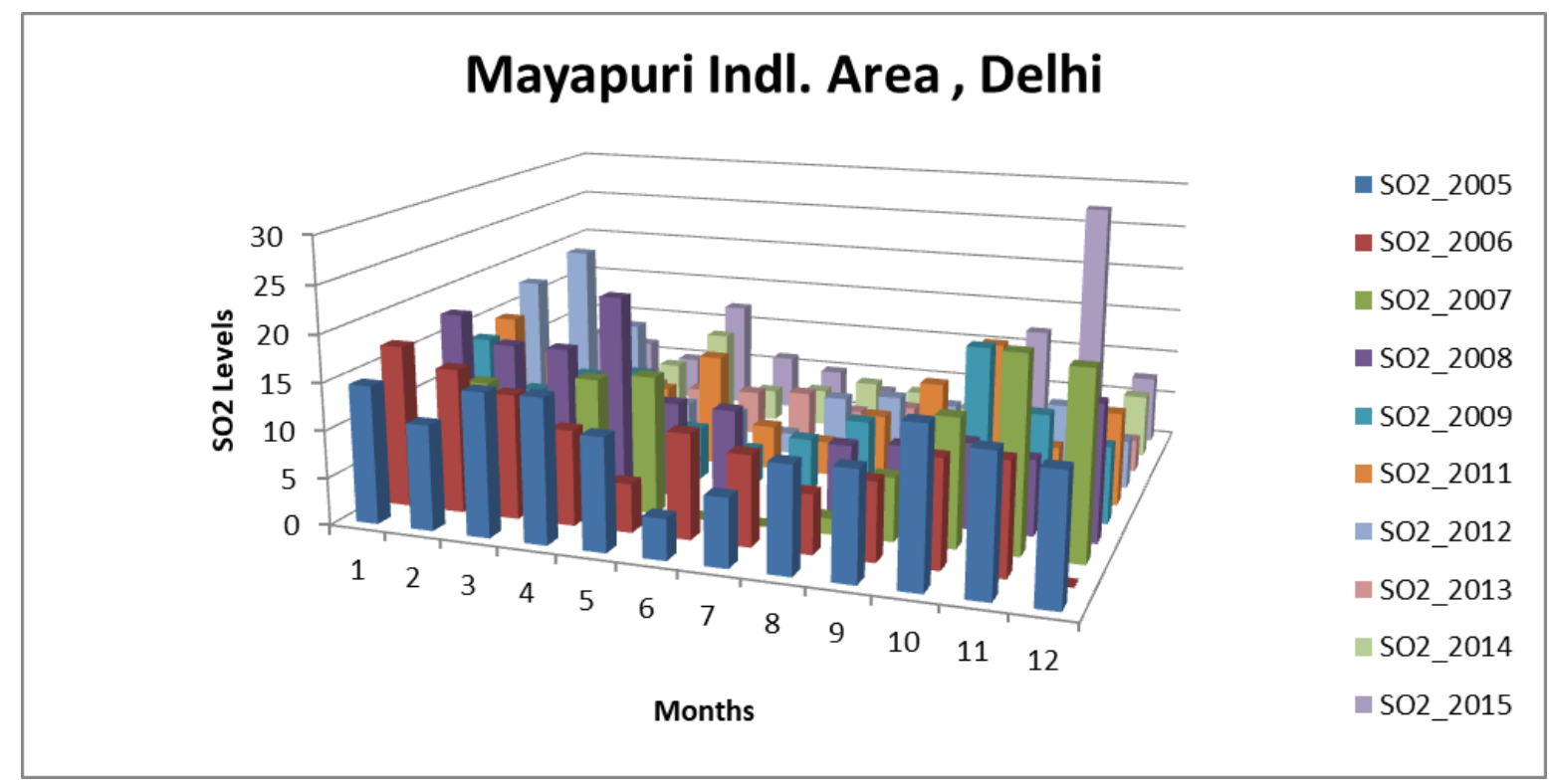

Figure 5: Variation in Sulphur Dioxide (SO2) level in Mayapuri Industrial Area, New Delhi from 2005 to 2015 as obtained from Kaggle

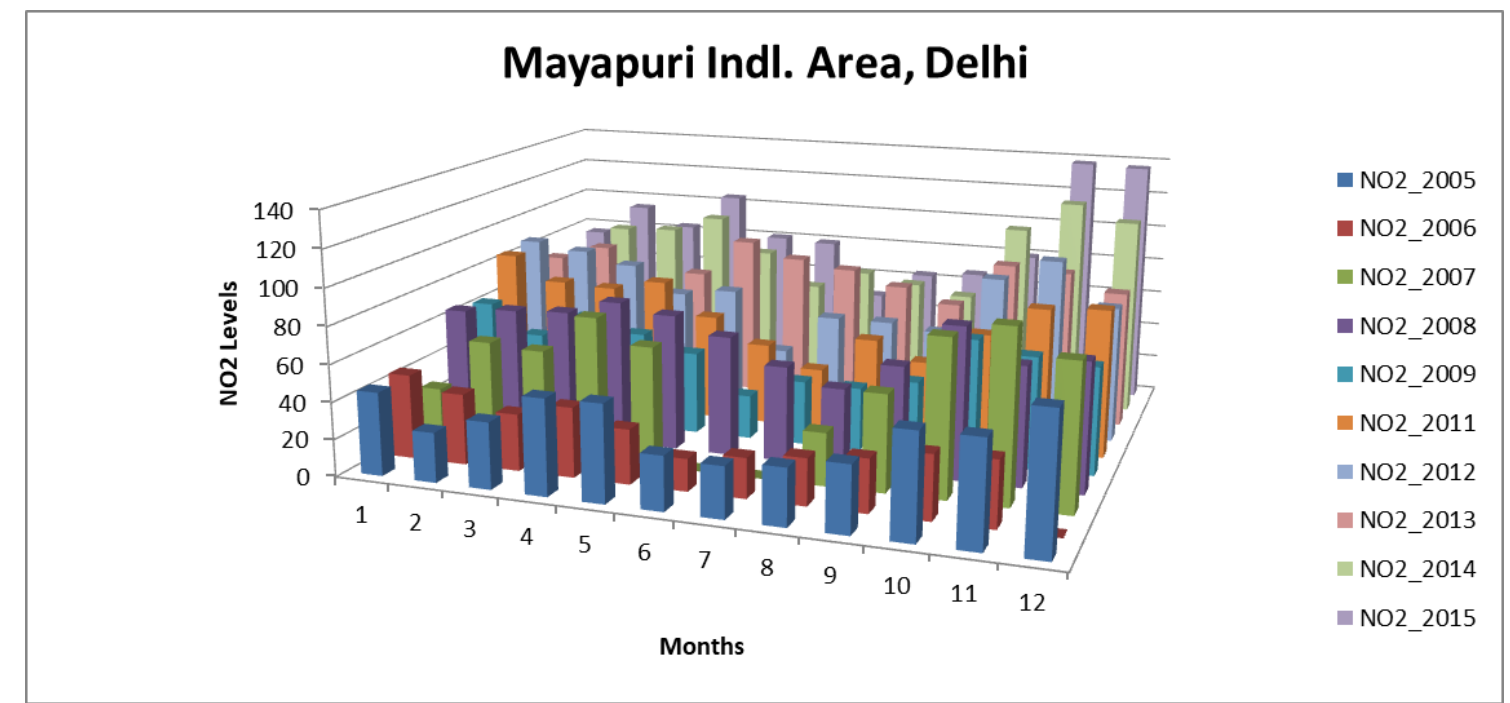

Figure 6: Variation in Nitrogen Dioxide (SO2) level in Mayapuri Industrial Area, New Delhi from 2005 to 2015 as obtained from Kaggle

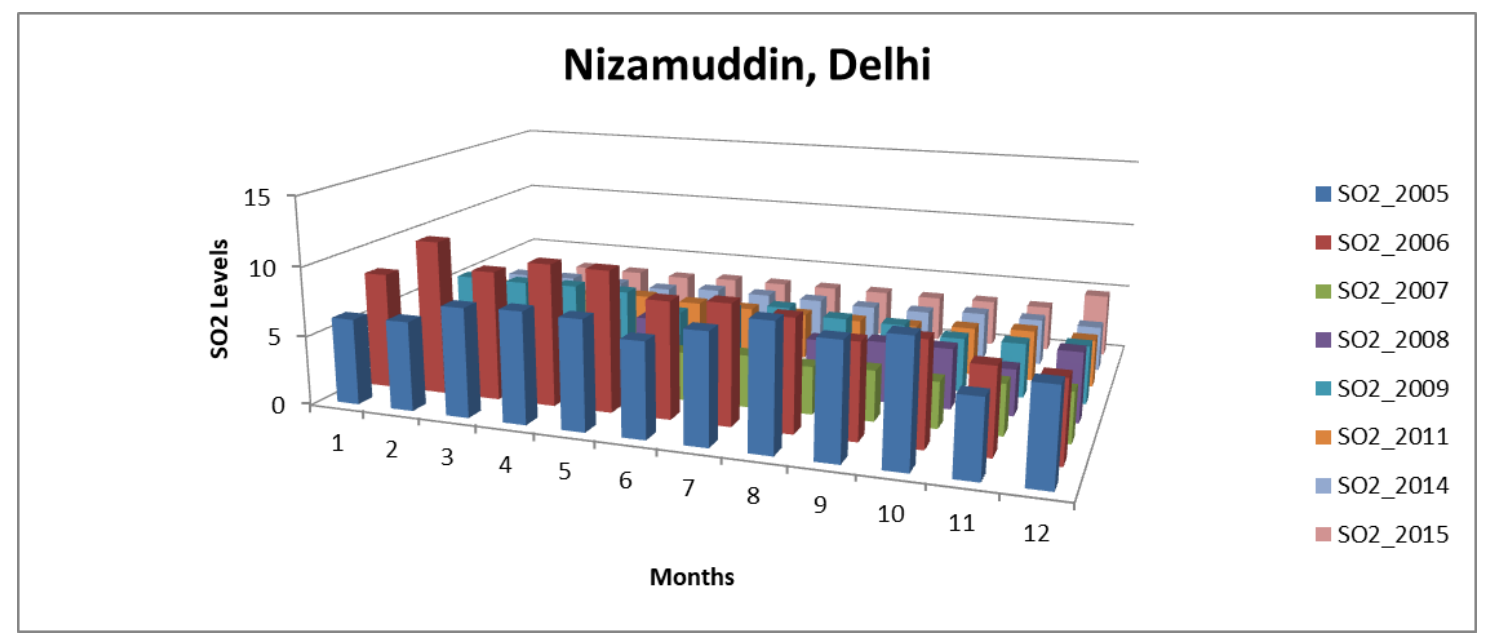

Figure 7: Variation in Sulphur Dioxide (SO2) level in Nizamuddin, New Delhi from 2005 to 2015 as obtained from Kaggle

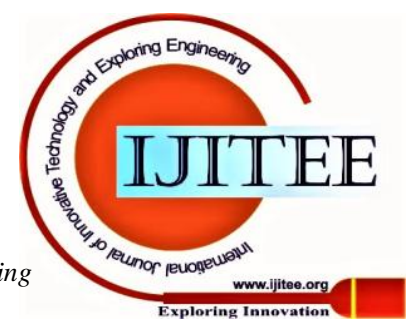




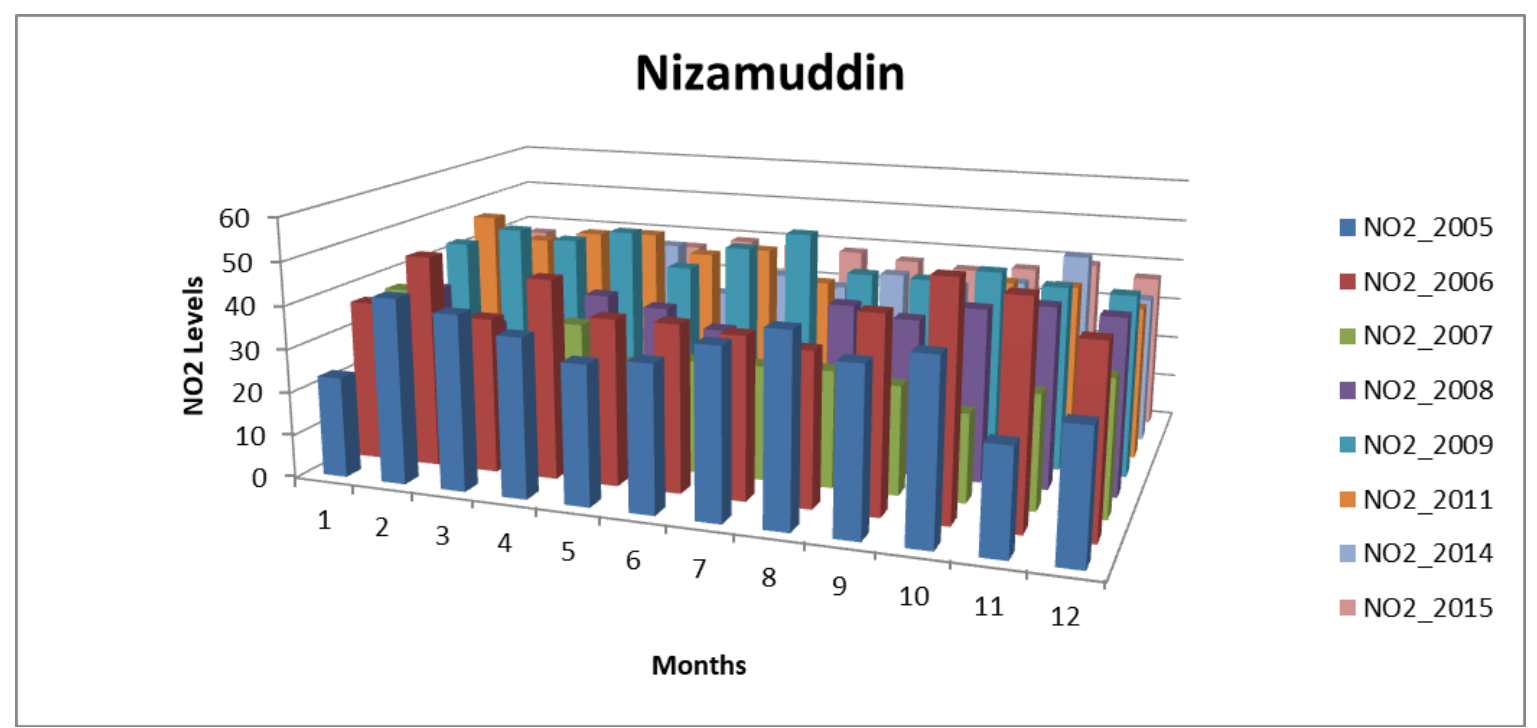

Figure 8: Variation in Nitrogen Dioxide (NO2) level in Mayapuri Industrial Area, New Delhi from 2005 to 2015 as obtained from Kaggle

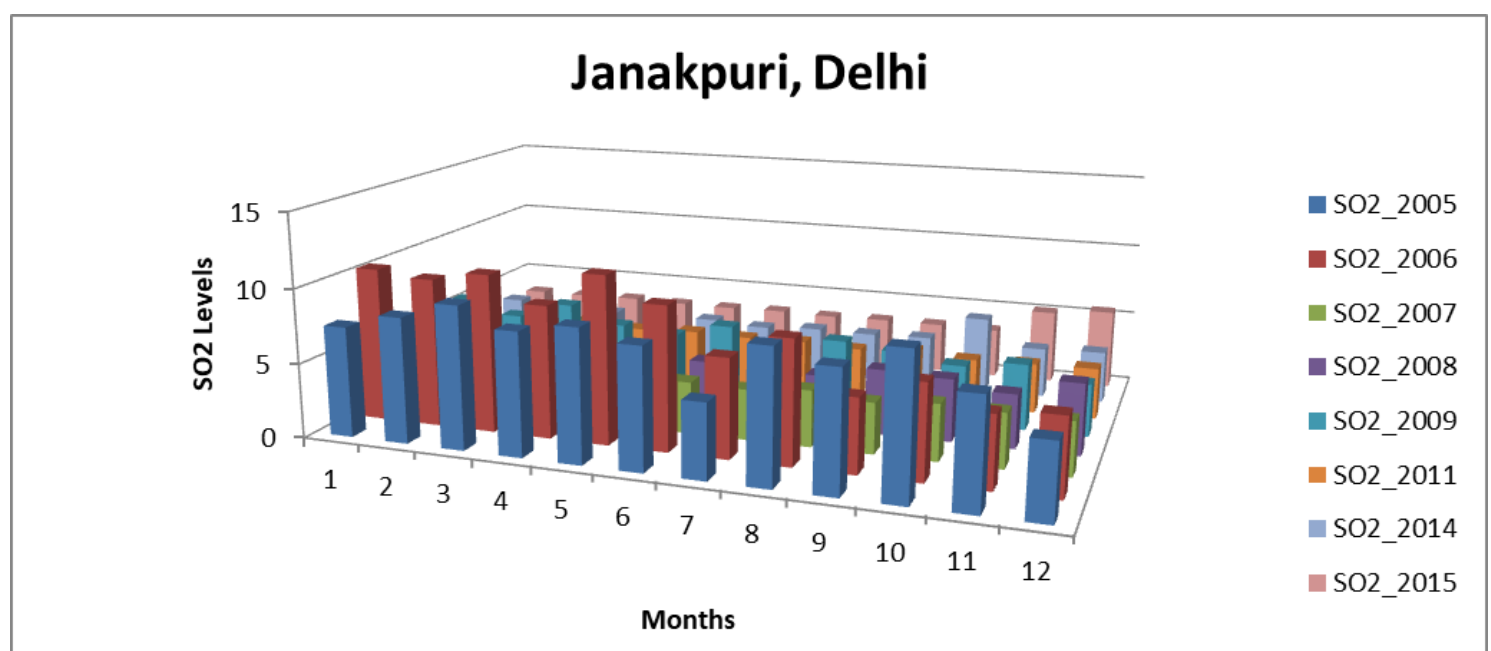

Figure 9: Variation in Sulphur Dioxide (SO2) level in Janakpuri, New Delhi from 2005 to 2015 as obtained from Kaggle

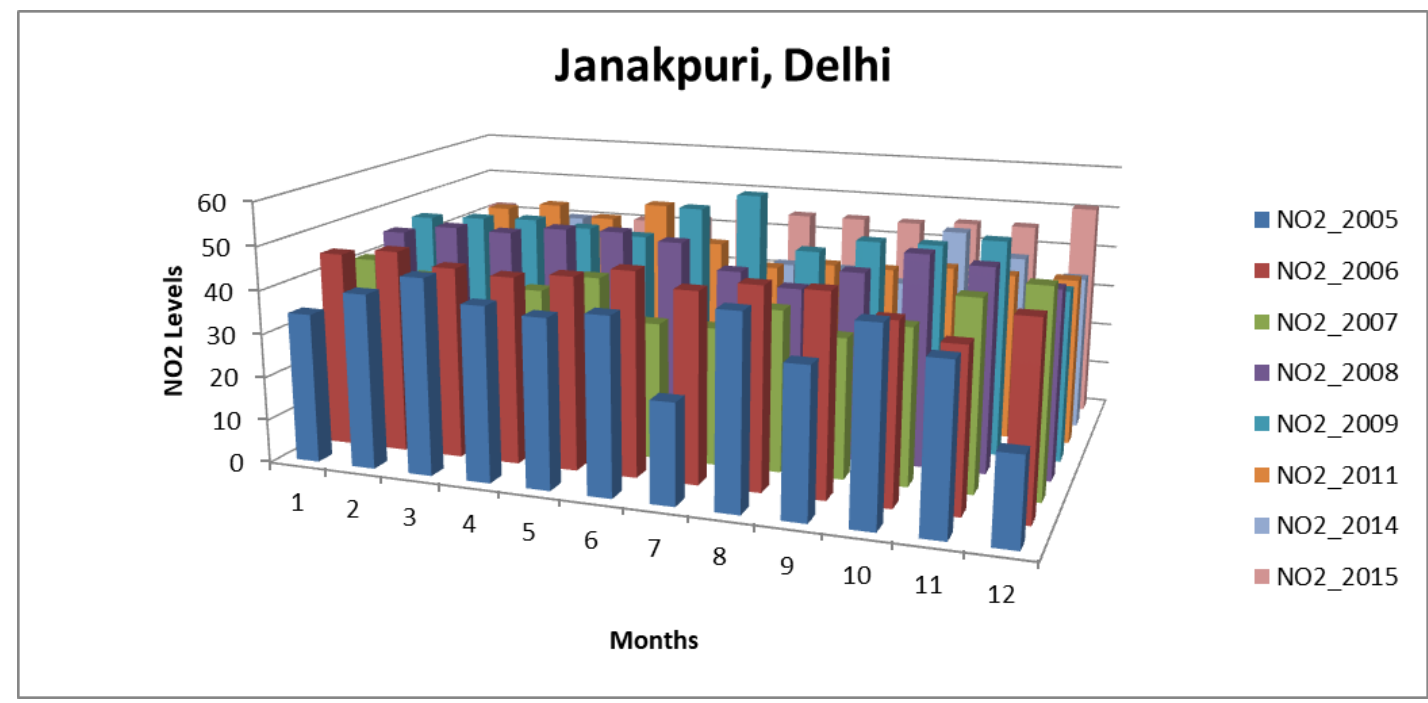

Figure 10: Variation in Nitrogen Dioxide (SO2) level in Janakpuri, New Delhi from 2005 to 2015 as obtained from Kaggle

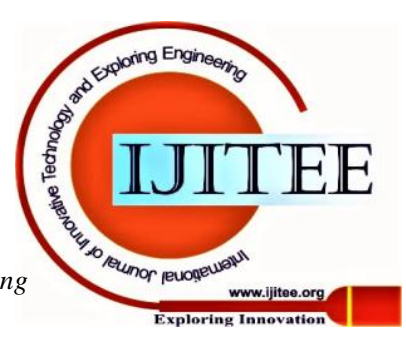


The various figures (3-10) depict continuous trend in rise and fall in levels ofpollutants like $\mathrm{NO} 2$ and $\mathrm{SO} 2$. It was found after careful scrutiny of all the graphs that level of $\mathrm{SO} 2$ has shown lesser variation in all the years. The level of SO2 in November and December in 2005 and 2015 has almost remained constant as shown in figure 9. For rest of the years in between 2005 and 2015 the level has seen a sharp decrease.

It is very much predictable that level of pollutant like NO2 will show continuous increase with increase in years in Inter State Bus Terminus (ISBT), Dehradun. This is due to immense burden on road transport cooperation due to increase in number traffic load. This scenario is very much evident from figure 4. activities. It houses more than 100 international as well as national corporate companies. Therefore a sudden spurt in traffic as well pollutants is very well expected as evident from figure 5 and figure 6 . The SO2 level has seen almost 2 fold increase 2005 to 2015.
Mayapuri Industrial area in New Delhi is a hub of business

Similarly the level of NO2 has seen sharp increase in levels as a continuous trend especially in the month of November and December as shown in figure 6.

Both NO2 and $\mathrm{SO} 2$ contribute in Smog formation [4,5]. Sulphur dioxide (SO2) reacts with oxidizing atmosphere produced by smog and contributes to stabilizing the smog. The level of both the gases therefore is not only responsible for deleterious health conditions. Sulphur dioxide influences human wellbeing when it is breathed in. It aggravates the nose, throat and also responsible for coughing and dyspnea. Sometimes it also affects respiratory system. The people with asthma face severe difficulties by exposure of such air. Nitrogen Oxide is also a harmful gaseous pollutant. Even the small level of NO2 is enough to create irritation in nose and throat. And high level can cause premature deaths. A person with longer exposure with $\mathrm{NO} 2$ is more susceptible of having asthma or more respiratory diseases. Thus these gaseous pollutants miserably affect the life of human being [8].

The SNS (seaborn pair plot) for Dehradun is shown in figure 11. The plot in figure 11 helps us in understating distribution of single variable and relationship between two variables.
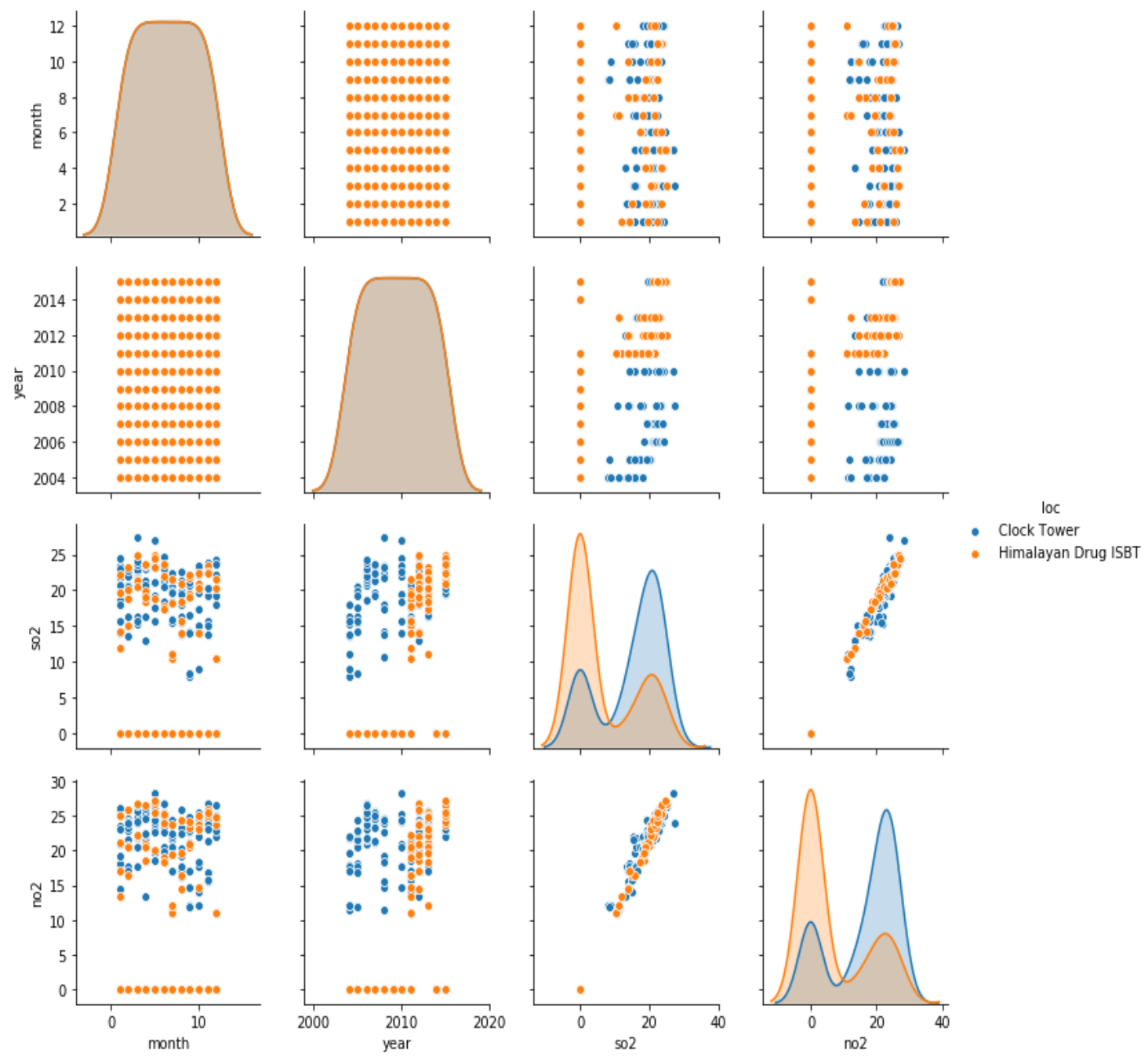

Figure 11: Pair plot for Dehradun.
As can be seen above the level of SO2 has been highest for Clock Tower in various months. The trend in the level of No2 has shown increase in ISBT during $2^{\text {nd }}, 3^{\text {rd }}$ and $4^{\text {th }}$ month. The relationship in between $\mathrm{NO} 2$ and $\mathrm{SO} 2$ shows a

major area occupied in ISBT and scattered area occupied in clock tower.

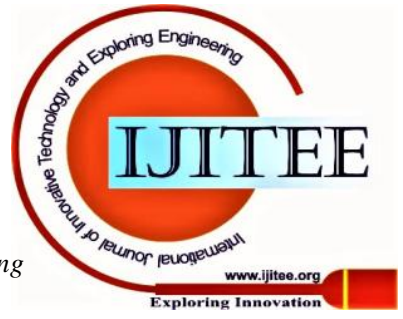


The density of both $\mathrm{SO} 2$ and $\mathrm{NO} 2$ is uniform in ISBT whereas distribution is scattered for both the gases in clock tower.

The pair plot for Delhi is shown in figure 12. The distribution of $\mathrm{NO} 2$ and $\mathrm{SO} 2$ shows concentration of $\mathrm{SO} 2$ and $\mathrm{NO} 2$ in Nizamuddin whereas both the gases are scattered in Mayapuri Industrial area. This is due to varying location of industries in Mayapuri.
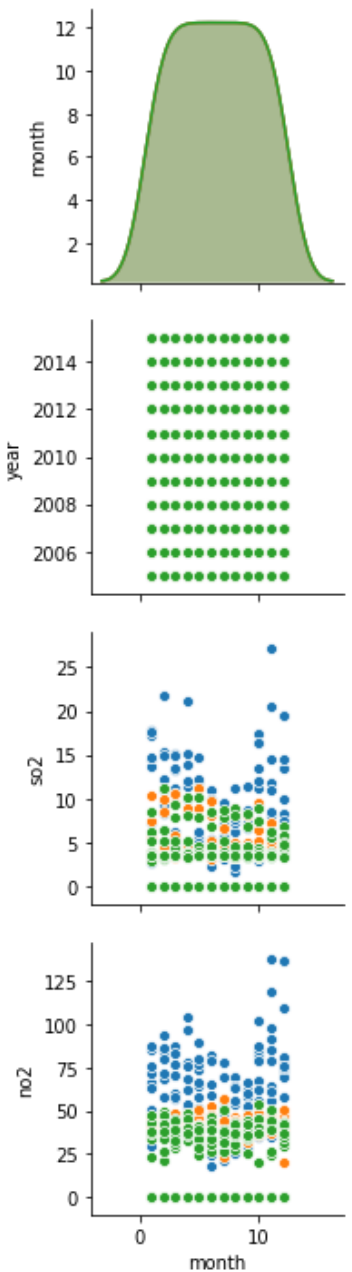
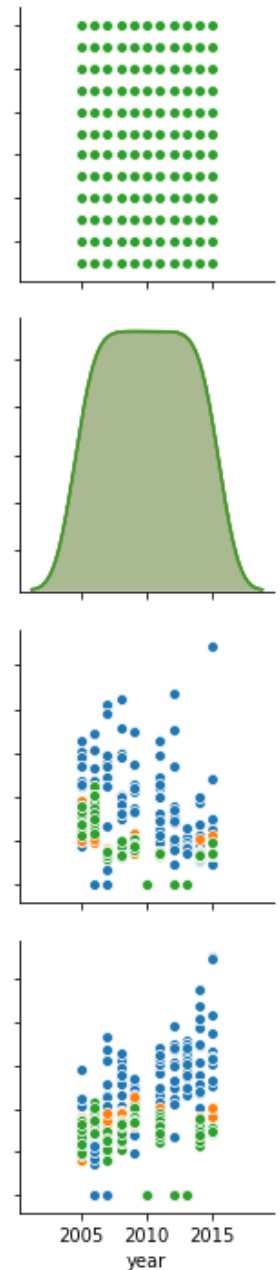
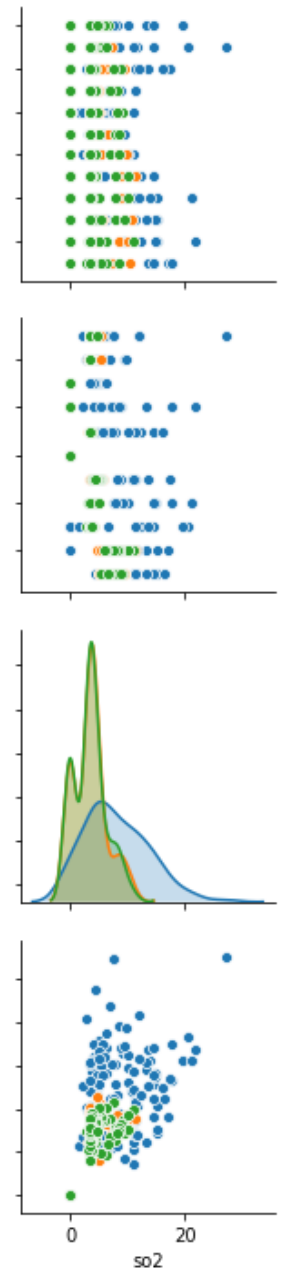
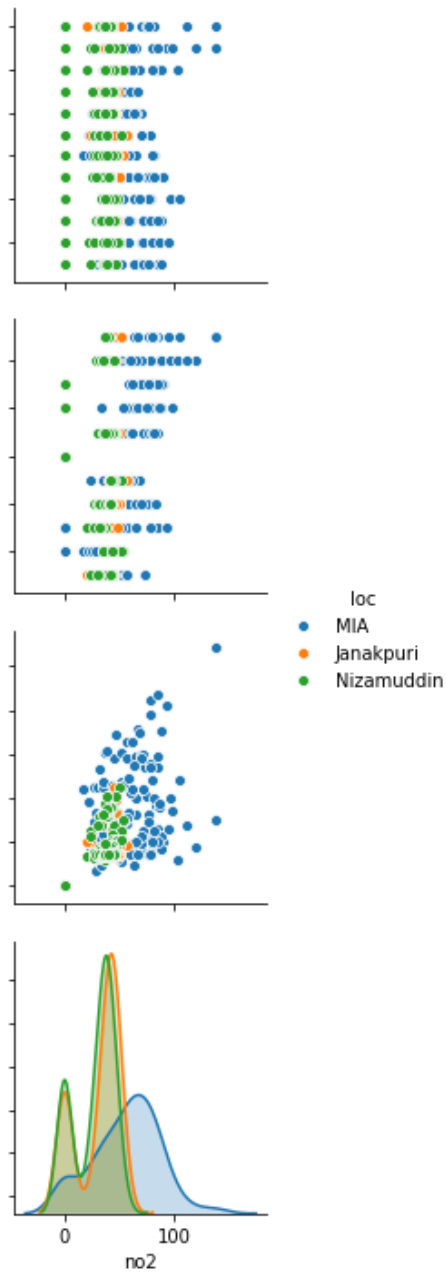

Figure 12: Pair plot for Delhi

\section{CONCLUSION}

This research paper helps in analyzing and understanding the trend in increase in various polluting gases with increase in urbanization and development. By scrutinizing the trend in variation in levels of gases a sensor system can be fitted at various places not only in Delhi and Dehradun but also in other metropolitan cities. The sensor will provide early indication of impending danger as well as help in planning and implementing various steps to curb these pollutants before level becomes high.

It is the need of the hour to analyze various data generated through latest technologies and implement the findings for benefit of the society on a whole.

\section{REFERENCES}

1. Air pollution at https://www.who.int/health-topics/airpollution\#tab=tab_1

2. Kaggle at https://www.kaggle.com/datasets

3. Clock tower, Dehradun at https://www.tourmyindia.com/states/uttarakhand/clock-towerdehradun.html.

4. Wm. E. Wilson Jr. , Arthur Levy \& D.B. Wimmer (1972) A Study of Sulfur Dioxide in Photochemical Smog, Journal of the Air Pollution
Control

Association, 10.1080/00022470.1972.10469605.

5. Nitrogen

dioxide

27-32,

DOI: https://www.environment.gov.au/protection/publications/factsheetnitrogen-dioxide-no2

6. Python at www.python.org.

7. Will Koehrsen (2018) Visualizing Data with Pairs Plots in Python, https://towardsdatascience.com/visualizing-data-with-pair-plots-inpython-f228cf529166.

8. https://towardsdatascience.com/india-air-pollution-data-analysis$\underline{\text { bd7dbfe93841 }}$ 